\title{
Superfluorescence of Photonic Paint
}

\author{
A. Yu. Zyuzin \\ A.F.Ioffe Physical-Technical Institute 194021 Saint-Petersburg, \\ Russia \\ and \\ Max-Planck-Institut für Festkörperforschung BP166, F-38042 \\ Grenoble, France
}

\begin{abstract}
We consider the cooperative decay of incoherently pumped atoms in a disordered medium, where light undergoes multiple scattering. It is shown that the cooperation number, which determines the duration and amplitude of superfluorescent impulses, is given by the number of atoms along a diffusive trajectory of the light propagating through the medium. We also consider the problem of reflection of a probe wave during cooperative emission.
\end{abstract}

\section{Introduction}

There is growing interest active photonic paints. These are media in which light undergoes multiple random scattering, resulting in a diffusive propagation of radiation, while interacting with atoms that can be pumped to obtain a positive population difference. The reflection and transmission of the electromagnetic waves through such a cavity has been extensively studied over the past decade. The speckle pattern resulting from scattering has an average enhancement in the direction opposite the direction of the incident radiation [1], (a comprehensive review of other statistical properties of the speckle of reflected and transmitted waves is given in [2]).

Feedback provided by scattering in such a random cavity can serve to set up laser oscillations [3]. The laser action in a powdered laser materials [ [4], 
laser dye solutions with scattering nanoparticles [6], and dye-doped microdroplets containing Intralipid as a scatterer [7] has recently been reported. These experiments concentrated mostly on temporal and spatial properties of emission.

Recently, the proposed [8] enhancement of the weak localization peak in backscattering from an amplifying photonic paint was observed [9].

The relevant question concerning recent observations of generation of light in active photonic paints [4] [5] is to what extent this phenomenon is reminiscent of superfluorescence [10] [11](i.e., the cooperative decay of an incoherently pumped system of dipole transitions, started by initial noise or an external electromagnetic field), which has usually been studied in systems without scattering.

Here we consider the cooperative decay of incoherently pumped atoms in a random cavity, which is a slab of thickness $L(L \gg l$, where $l$ is the mean free path of radiation). This geometry is often used in experiments. The time that light spends in this cavity is of order $\frac{L^{2}}{D}$, where $D$ is diffusion constant. This time is to be compared with the energy exchange time between atoms and field. We show that if the latter is greater than $\frac{L^{2}}{D}$, then after some delay, the system will generate a superfluorescent pulse of hyperbolic secant form.

The duration of the superfluorescent pulse is $\tau_{\text {rad }} N_{C}^{-1}$ [10], where $\tau_{\text {rad }}$ is the time of radiative decay of a single atom and $N_{C}$ is the cooperation number, i.e., the number of atoms that take part in cooperative decay. We find that in disordered systems, this number is $N_{C} \sim \rho \frac{\lambda^{2} L^{2}}{l}(\lambda$ is the wavelength of the radiation and $\rho$ is the density of active atoms, such that $\rho \lambda^{3} \gg 1$ ), i.e., it is equal to the number of atoms in a tube with cross section $\lambda^{2}$ and length of the order of $\frac{L^{2}}{l}$, which is the length of a diffusive trajectory of radiation.

The intensity of radiation of cooperating atoms at the maximum of the superfluorescent pulse is $\sim \frac{N_{C}^{2}}{\tau_{\text {rad }}}$ [10. We show that the diffusive slab radiates at maximum as a system of $\frac{V}{N_{C}}$ independent groups of cooperating atoms, and at the peak of superfluorescent pulse, the intensity emitted by the slab is $\sim \frac{N_{C}^{2}}{\tau_{\text {rad }}} \times \frac{V}{N_{C}} . V$ is the volume of the slab.

The maximum cooperation number for given $\tau_{\text {rad }}$ and density of active atoms is determined by the condition that the time of energy exchange between atoms and the field equals the time that light spends in the cavity. From this condition, we find that the maximum cooperation number in the random cavity is $N_{C}^{\max } \sim \lambda \sqrt{c \rho \tau_{\text {rad }}}$, where $c$ is the speed of radiation in the 
slab.

These results are valid in the case of weak dephasing processes and long relaxation of population difference. Below we take into account the effect of dephasing on superfluorescence.

In the limit of the large escape time $\frac{L^{2}}{D}$ of radiation, atoms exchange energy with the field many times, so stimulated emission becomes important and the system exhibits oscillatory behavior.

We also consider the reflection of the probe wave during decay of the pumped system.

\section{Basic equations.}

We model a random medium in the following way. The dielectric function $\epsilon(\vec{r})$ of the medium, which contains active atoms, is a random function of position, such that $\langle\epsilon(\vec{r})\rangle$. Scattering of light is due to fluctuations of the dielectric function with white-noise like variance $\left\langle\delta \epsilon(\vec{r}) \delta \epsilon\left(\overrightarrow{r^{\prime}}\right)\right\rangle=$ $\frac{\lambda^{4}}{4 \pi^{3} l} \delta\left(\vec{r}-\overrightarrow{r^{\prime}}\right)$.

We consider the case of a weakly disordered system $l \gg \lambda$, with dimensions larger the than mean free path, so propagation of the field can be described as a diffusion process with diffusion constant $D=\frac{c l}{3}, c$ is the speed of light in the medium.

The coupling between the polarization density $\frac{1}{2}\left\{e^{i \omega t} P(\vec{r} ; t)+e^{-i \omega t} P^{*}(\vec{r} ; t)\right\}$, averaged over scales smaller than $\lambda$, the population difference density $\Delta N(\vec{r} ; t)$, and the field $\frac{1}{2}\left\{e^{i \omega t} E(\vec{r} ; t)+e^{-i \omega t} E^{*}(\vec{r} ; t)\right\}$ can be described by the classical Maxwell-Bloch equations. In this approach, amplified spontaneous emission noise is neglected, which is a good approximation for superfluorescence [?]. $P(\vec{r} ; t)$ and $E(\vec{r} ; t)$ are slowly time-varying complex quantities, which we consider to be scalars; $\omega$ is the atomic frequency.

First two Maxwell-Bloch equations have the form [13].

$$
\begin{gathered}
{\left[\frac{d}{d t}+\gamma\right] P(\vec{r} ; t)=\frac{i|\mu|^{2}}{\hbar} \Delta N(\vec{r} ; t) E(\vec{r} ; t)} \\
\frac{d}{d t} \Delta N(\vec{r} ; t)=-\frac{i}{2 \hbar}\left\{P^{*}(\vec{r} ; t) E(\vec{r} ; t)-P(\vec{r} ; t) E *(\vec{r} ; t)\right\}
\end{gathered}
$$

Here $\gamma$ is the inverse dephasing time and $\mu$ is the electric dipole moment. 
It is assumed that the population inversion relaxation time is longer than the delay time of the superfuorescent pulse. We also neglect inhomogeneous broadening.

The quantities $\Delta N(\vec{r} ; t)$ and $\frac{P\left(\vec{r}_{;} ; t\right)}{\mu}$ are components of the local Bloch vector averaged over scales smaller than the wavelength of the radiation. The rate at which its length decreases, according to (1) and (2), is determined by $\gamma^{-1}$.

The field wave equation for the slow time-varying component $E(\vec{r} ; t)$ has the form

$$
i \frac{d E(\vec{r} ; t)}{d t}-\left\{-\frac{c^{2}}{2 \omega} \Delta-\frac{\omega \epsilon(\vec{r})}{2}\right\} E(\vec{r} ; t)=2 \pi \omega P(\vec{r} ; t)
$$

Although $E(\vec{r} ; t)$ and $P(\vec{r} ; t)$ vary slowly in time, they still contain spatial random phases, which result from random interference between waves coming to the point $\vec{r}$ via different diffusive trajectories. To get rid of these phase factors, it is convenient to consider the diffusion propagator $D\left(\vec{r} ; t_{1}, t_{2}\right)$, which determines the correlation function of the polarization density and field :

$$
\left\langle E\left(\vec{r} ; t_{1}\right) E^{*}\left(\vec{r} ; t_{2}\right)\right\rangle=4 \pi k^{3} \omega D\left(\vec{r} ; t_{1}, t_{2}\right)
$$

Correlation functions involving the polarization density can be obtained by using Eq.(1).

To obtain the equation for the diffusion propagator, it is convenient to eliminate the polarization density from Eqs.(1) and (3). Then the usual diagram technique [14 makes it possible to calculate the average of the product $E\left(\vec{r} ; t_{1}\right) E^{*}\left(\vec{r} ; t_{2}\right)$.

Considering the evolution of the Bloch vector from time $t=0$, at which a positive population difference is created, we obtain for the diffusion propagator

$$
\begin{gathered}
\left\{\frac{d}{d t_{1}}+\frac{d}{d t_{2}}-D \vec{\nabla}^{2}\right\} D\left(\vec{r} ; t_{1}, t_{2}\right)=f\left(\vec{r} ; t_{1}, t_{2}\right)+ \\
+\frac{1}{\rho \tau_{0}^{2}} \int_{0}^{t_{1}} d t \exp \left\{-\gamma\left(t_{1}-t\right)\right\} \Delta N(\vec{r}, t) D\left(\vec{r} ; t, t_{2}\right)+
\end{gathered}
$$




$$
+\frac{1}{\rho \tau_{0}^{2}} \int_{0}^{t_{2}} d t \exp \left\{-\gamma\left(t_{2}-t\right)\right\} \Delta N(\vec{r}, t) D\left(\vec{r} ; t_{1}, t_{2}\right)
$$

Here $\tau_{0}=\sqrt{\frac{\hbar}{2 \pi \rho \omega|\mu|^{2}}}=\sqrt{\frac{4 \pi \tau_{\text {rad }}}{3 \omega \rho \lambda^{3}}}$ is the characteristic time of energy exchange between the field and the atomic system [?], $\rho$ is the density of active atoms, and $\tau_{\text {rad }}^{-1}=\frac{8 \pi^{2}|\mu|^{2}}{3 \hbar \lambda^{3}}$ is the radiative decay time of a single atom.

The function $f\left(\vec{r} ; t_{1}, t_{2}\right)$ depends on initial conditions. Here we choose the initial condition such that $\langle P(\vec{r}, t=0)\rangle=0$ and $\left\langle P(\vec{r}, t=0) P^{*}\left(\overrightarrow{r^{\prime}}, t=0\right)\right\rangle=$ $\rho|\mu|^{2} \delta\left(\vec{r}-\overrightarrow{r^{\prime}}\right)$. This initial condition corresponds to an initial incoherent state. In this case

$$
f\left(\vec{r} ; t_{1}, t_{2}\right)=\rho|\mu|^{2} \exp \left\{-\gamma\left(t_{1}+t_{2}\right)\right\}
$$

for times greater than the mean free time of radiation $\frac{l}{c}$.

The equation for the mean population inversion density can be obtained by using Eqs. (1), (2) and (5):

$\frac{d \Delta N(\vec{r}, t)}{d t}=-\frac{(2 \pi)^{3}}{\hbar \rho \lambda^{3} \tau_{0}^{2}} \int_{0}^{t} d t \exp \left\{-\gamma\left(t-t^{\prime}\right)\right\} \Delta N\left(\vec{r}, t^{\prime}\right)\left\{D\left(\vec{r} ; t^{\prime}, t\right)+D\left(\vec{r} ; t, t^{\prime}\right)\right\}$

For the population difference we choose $\Delta N(\vec{r}, t=0)=\Delta N>0$ as the initial condition $(\Delta N=\rho)$.

The usual boundary conditions for the diffusion propagator are $D\left(\vec{r} ; t_{1}, t_{2}\right)=$ 0 on an open surface and $\vec{n} \vec{\nabla} D\left(\vec{r} ; t_{1}, t_{2}\right)=0$ on a reflecting surface; $\vec{n}$ is normal to the reflecting surface.

The diffusion approach is justified if the time of energy exchange between atoms and field is greater than mean the free time of radiation, $\tau_{0} \gg \frac{l}{c}$.

\section{Cooperative decay in photonic paint}

Below we consider a slab of thickness $L(L \gg l)$. Let $z$ be the coordinate across the slab $L \succeq z \succeq 0$. It is convenient to study the solution of Eq. (5) in the form 


$$
D\left(\vec{r} ; t_{1}, t_{2}\right)=\sqrt{\frac{L}{2}} \sum_{n=1}^{\infty} \Psi_{n}(z) D_{n}\left(t_{1}, t_{2}\right)
$$

where $\Psi_{n}(z)=\sqrt{\frac{2}{L}} \sin \frac{\pi n z}{L}$ is an eigenfunction of the diffusion equation with boundary condition $\Psi_{n}(z)=0$ at the free boundaries $z=0, L$.

Let us consider the initial evolution of the diffusion propagator, when the population difference does not depend on time. For the coefficients in (8), we obtain from (5)

$$
\begin{aligned}
\left\{\frac{d}{d t_{1}}+\frac{d}{d t_{2}}+\omega_{n}\right\} D_{n}\left(t_{1}, t_{2}\right)= & \sqrt{\frac{2}{L}} \rho|\mu|^{2} \int d z \Psi_{n}(z) \exp \left\{-\gamma\left(t_{1}+t_{2}\right)\right\}+ \\
& +\frac{\Delta N}{\rho \tau_{0}^{2}} \int_{0}^{t_{1}} d t \exp \left\{-\gamma\left(t_{1}-t\right)\right\} D_{n}\left(t, t_{2}\right)+(9) \\
& +\frac{\Delta N}{\rho \tau_{0}^{2}} \int_{0}^{t_{2}} d t \exp \left\{-\gamma\left(t_{2}-t\right)\right\} D_{n}\left(t_{1}, t_{2}\right)
\end{aligned}
$$

Here $\omega_{n}=\frac{D \pi^{2} n^{2}}{L^{2}}$ is an eigenvalue of the diffusion equation. Solving Eq. (9) via the Laplace transform with initial conditions $D_{n}\left(0, t_{2}\right)=D_{n}\left(t_{1}, 0\right)=$ 0 (the field vanishes at $t=0$ ), we obtain

$$
D_{n}(t, t) \sim \exp \left\{\left(\sqrt{\left(\frac{\omega_{n}}{2}-\gamma\right)^{2}+\frac{4 \Delta N}{\rho \tau_{0}^{2}}}-\frac{\omega_{n}}{2}-\gamma\right) t\right\}
$$

The critical value of positive inversion density $\Delta N_{n}$, above which the growth rate of a particular diffusion mode $Z_{n}=\sqrt{\left(\frac{\omega_{n}}{2}-\gamma\right)^{2}+\frac{4 \Delta N}{\rho \tau_{0}^{2}}}-\frac{\omega_{n}}{2}-\gamma$ becomes positive, is $\Delta N_{n}=\frac{\omega_{n} \gamma}{2} \rho \tau_{0}^{2}[?]$. More detailed calculations of (10) are given in the next section.

To proceed further in solving Eqs. (5) and (7), we make two approximations.

1). Below we consider the case of fast escape of radiation from the system, where $\omega_{1} \gg \frac{d}{d t_{1}}, \frac{d}{d t_{2}}, \gamma$ (or, according to (10), $\tau_{0} \omega_{1}>1$ for weak dephasing), so we can neglect the time derivative in Eq. (5). In the language of superfluorescence, this situation corresponds to the case in which there is no energy exchange between the emitted field and atomic subsystem [?]. The field serves only to develop correlation between atoms. 
2). We consider only the most unstable mode $D_{1}\left(t_{1}, t_{2}\right)$. At $t=0$, the off-diagonal elements of $\Delta N_{n m} \equiv \int d z \Delta N(z, t) \Psi_{n}(z) \Psi_{m}(z)$ are zero by definition, and interaction between modes is irrelevant for most of the time of decay. We therefore assume that the interaction of the first diffusion mode $D_{1}\left(t_{1}, t_{2}\right)$ with higher modes does not qualitatively change the description of cooperative decay.

Under these assumptions the equation for the diffusion propagator has the form

$$
\begin{aligned}
D_{1}\left(t_{1}, t_{2}\right)= & \frac{4}{\pi \omega_{1}} \rho|\mu|^{2} \exp \left\{-\gamma\left(t_{1}+t_{2}\right)\right\}+ \\
& +\frac{1}{\rho \omega_{1} \tau_{0}^{2}} \int_{0}^{t_{1}} d t \exp \left\{-\gamma\left(t_{1}-t\right)\right\} \Delta N_{11}(t) D_{1}\left(t, t_{2}\right)+ \\
& +\frac{1}{\rho \omega_{1} \tau_{0}^{2}} \int_{0}^{t_{2}} d t \exp \left\{-\gamma\left(t_{2}-t\right)\right\} \Delta N_{11}(t) D_{1}\left(t_{1}, t_{2}\right)
\end{aligned}
$$

and for the population difference

$$
\frac{d}{d t} \Delta N_{11}(t)=-\frac{k^{3}}{\hbar}\left\{\frac{8}{3 \pi} \omega_{1} D_{1}(t, t)-\rho|\mu|^{2} \exp \{-2 \gamma t\}\right\}
$$

Introducing

$$
D_{1}\left(t_{1}, t_{2}\right) \equiv \exp \left\{-\gamma\left(t_{1}+t_{2}\right)\right\} G\left(\chi\left(t_{1}\right) ; \chi\left(t_{2}\right)\right)
$$

in (11),where

$$
\chi(t)=\frac{1}{\rho \omega_{1} \tau_{0}^{2}} \int_{0}^{t} d t \Delta N_{11}(t)
$$

we obtain

$$
G\left(\chi_{1} ; \chi_{2}\right)=\frac{4}{\pi \omega_{1}} \rho|\mu|^{2}+\int_{0}^{\chi_{1}} d \chi G\left(\chi ; \chi_{2}\right)+\int_{0}^{\chi_{2}} d \chi G\left(\chi_{1} ; \chi\right)
$$

Equation (15) can be solved by a Laplace transform as 


$$
G\left(\chi_{1} ; \chi_{2}\right)=\frac{4 \rho|\mu|^{2}}{\pi \omega_{1}} \int_{C-i \infty}^{C+i \infty} \frac{d z_{1}}{2 \pi i} \frac{d z_{2}}{2 \pi i} \frac{\exp \left\{z_{1} \chi_{1}+z_{2} \chi_{2}\right\}}{z_{1} z_{2}-z_{1}-z_{2}}
$$

The asymptotic form of (16) for $\chi_{1}=\chi_{2} \equiv \chi>1$ is

$$
G(\chi ; \chi) \simeq \frac{2 \rho|\mu|^{2}}{\pi \omega_{1}} \frac{\exp \{4 \chi\}}{\sqrt{\pi \chi}}
$$

The equation for the population difference $(\chi>1)$ is

$$
\frac{d^{2}}{d t^{2}} \chi=-\frac{8 k^{3}}{3 \pi \hbar \rho \tau_{0}^{2}} G(\chi ; \chi) \exp \{-2 \gamma t\}
$$

Taking into account only exponential factors, we obtain the solution of Eq. (18) :

$$
\Delta N_{11}(t)=\delta N \tanh \left\{\frac{2 \delta N\left(t_{0}-t\right)}{\rho \tau_{0}^{2} \omega_{1}}\right\}+\frac{\gamma \rho \tau_{0}^{2} \omega_{1}}{2}
$$

Here we introduce $\delta N=\Delta N-\frac{\gamma \rho \tau_{0}^{2} \omega_{1}}{2} ; \Delta N$ is the population difference at the beginning of exponential growth of radiative intensity, when deviation from the initial population difference is small $(\Delta N=\rho)$.

The delay time in (19) is $t_{0}=\frac{\rho \tau_{0}^{2} \omega_{1}}{2 \delta N} \ln \left\{\frac{\delta N}{\rho} \sqrt{\rho \lambda^{3}} \frac{L}{\sqrt{l \lambda}}\right\}$. In deriving this expression we took into account the relation between the time of energy exchange between atoms and field and $|\mu|$, which enters into the initial condition for polarization density.

The radiative intensity is proportional to $\frac{d \Delta N_{11}}{d t}$, and is emitted as a hyperbolic-secant pulse. The result (19) coincides with that of the Markov theory of superfluorescence in a system without scattering [10, ?]. The difference is in definition of the cooperation number.

It follows from Eq. (19) that in the case of weak dephasing, the duration of a superfluorescent pulse is $\frac{\tau_{0}^{2} \omega_{1}}{4} \equiv \tau_{\text {rad }} N_{C}^{-1}$, where $\tau_{\text {rad }}$ is the time of radiative decay of a single atom and $N_{C}=\frac{18 \rho \lambda^{2} L^{2}}{\pi^{2} l}$ is the cooperation number, i.e., the number of atoms that take part in the cooperative decay. This is equal to the number of atoms in a tube of cross section $\lambda^{2}$ with the length of the diffusive trajectory $\frac{L^{2}}{l}$. The maximum of the cooperation number is determined by the condition $\tau_{0} \omega_{1}=1$, whereupon $N_{C}^{\max }=2 \lambda \sqrt{6 c \rho \tau_{\text {rad }}}$. Under this condition, atoms can exchange energy with the field only once, i.e., stimulated emission 
can be neglected. We note that for a given density $\rho$, decay time $\tau_{\text {rad }}$, and velocity, the maximum cooperation number in a disordered system is smaller, than in a pencil-shaped system without scattering [15].

The maximum emitted radiation is $V \frac{d}{d t} \Delta N_{11}\left(t=t_{0}\right)$ ( $V$ is the volume of the slab). It can also be written $\mathrm{N} \times \frac{N_{C}^{2}}{\tau_{\text {rad }}}$, where $\mathrm{N}=\frac{V \rho}{N_{C}}$ is the number of cooperative regions in the slab. The cooperative decay in a diffusive medium can therefore be interpreted as the independent cooperative decay of $\mathrm{N}=\frac{V \rho}{N_{C}}$ systems, each consisting of $N_{C}$ atoms.

Dephasing processes increase the duration of a pulse by the factor $\frac{\rho}{\Delta N-\frac{\gamma \rho \tau_{0}^{2} \omega_{1}}{2}}$, and decrease the peak intensity by the square of this factor. Note that this result coincides with that for a system without scattering [?].

If $1 \gg \tau_{0} \omega_{1}$, atoms exchange energy with the field many times. In this case we expect spiking of intensity. The frequency of spiking can be estimated [13 from Eq. (10) as $\sqrt{\left|\frac{4 \Delta N}{\rho \tau_{0}^{2}}\right|-\left(\frac{\omega_{1}}{2}-\gamma\right)^{2}}$. To obtain this expression we insert a negative value of the population inversion [13] into (10) (this situation will occur after the pumped atoms exchange energy with the field).

\section{Amplification in the backward direction}

Correlation between pumped atoms can also be due to the external field, which stimulates emission in the forward direction in a system without scattering [13]. In a disordered system one might expect enhancement of emission in the backward direction.

Here we consider the reflection of a weak probe plane wave with frequency during the development of superfluorescent emission. The amplitude of the probe is low, so the effect of the external field on emission can be neglected. We can also neglect interference between the external field with the emitted one, because the initial state of polarization is incoherent. Below we consider in detail the linear stage of decay when the inversion density is high enough to produce only the lowest diffusion mode instability, $\Delta N=\Delta N_{1}(1+\delta)$, $\delta \ll 1$. This situation resembles the experimental setup of [9].

It is convenient to calculate the albedo, which is the ratio between the intensities of the reflected and incident fields. The time-dependent albedo can be expressed as 17] 
$\alpha(\vec{q} ; t)=\frac{c}{4 \pi l^{2}} \int_{0}^{\infty} d z d z^{\prime} \exp \left\{-\frac{z+z^{\prime}}{l}\right\} \int d \vec{\rho}\{1+\cos \vec{q} \vec{\rho}\} D\left(z, z^{\prime}, \vec{\rho} ; t, t\right)$

Here $\vec{q}$ is the sum of the incident and outgoing wave vectors, and $\vec{\rho}$ is the position in the plane. Diffusion propagator (20) obeys Eq. (5) with the substitution of $\delta\left(\vec{r}-\overrightarrow{r^{\prime}}\right)$ for $f\left(\vec{r} ; t_{1}, t_{2}\right)$. We also assume that the incident wave is close to the normal to the surface.

The first term describes diffusion scattering, and the second term describes the interference part, which is strongly peaked in the backward direction. The physical mechanism of the interference contribution is exhaustively discussed in the literature; see, for example Refs.. [1, 8] and references therein.

The diffusion propagator can be represented as

$$
D\left(\vec{r}, \overrightarrow{r^{\prime}} ; t_{1}, t_{2}\right)=\sum_{n} \Psi_{n}(z) \Psi_{n}\left(z^{\prime}\right) \exp \left\{i \vec{q}\left(\vec{\rho}-\overrightarrow{\rho^{\prime}}\right)\right\} \hat{D_{n}}\left(q ; t_{1}, t_{2}\right)
$$

The Laplace transform of Eq. (5) for time-independent $\Delta N>0$ yields

$$
\hat{D_{n}}\left(q ; t_{1}, t_{2}\right)=\int_{-i \infty+C}^{i \infty+C} \frac{d p_{1} d p_{2}}{(2 \pi i)^{2}} \frac{\exp \left(p_{1} t_{1}+p_{2} t_{2}\right)}{p_{1} p_{2}\left\{p_{1}+p_{2}+\Omega_{n}(q)-\frac{\Delta N}{\rho \tau_{0}^{2}}\left(\frac{1}{p_{1}+\gamma}+\frac{1}{p_{2}+\gamma}\right)\right\}}
$$

where the real part of the integration contour passes to the left of all singularities, and $\Omega_{n}(q)=D q^{2}+\omega_{n}$ is the eigenvalue of the diffusion equation for the slab geometry.

At $t_{1}=t_{2}$, integrating over the difference $p_{1}-p_{2}$ in (22), we obtain for the first mode at $t>\omega_{1}^{-1}$

$$
\hat{D}_{n}(q ; t, t)=\frac{(2 \gamma)^{\frac{3}{2}}}{\sqrt{\omega_{1}\left(\omega_{1}+2 \gamma\right)}} \int_{-i \infty+C}^{i \infty+C} \frac{d p}{2 \pi i} \frac{\exp p t}{p \sqrt{p-Z_{1}(q)}\left(p+\sqrt{\frac{\gamma\left(\omega_{1}+2 \gamma\right)}{2 \omega_{1}}} \sqrt{p-Z_{1}(q)}\right)}
$$
$\hat{D_{n}}(q ; t, t)$.

Here we introduce $Z_{1}(q)=\frac{2 \gamma \omega_{1}}{\omega_{1}+2 \gamma}\left(\delta-\frac{D q^{2}}{\omega_{1}}\right)$, which is the growth rate of 
For moderate times $\frac{t \omega_{1} Z_{1}^{2}}{\gamma\left(\omega_{1}+2 \gamma\right)}<1$, we obtain from $(23)$

$$
\hat{D}_{n}(q ; t, t)=\frac{2\left(\exp \left\{Z_{1}(q) t\right\}-1\right)}{\omega_{1}\left\{\delta-\frac{D q^{2}}{\omega_{1}}\right\}}
$$

This expression is valid for either sign of $Z_{1}$, i.e., above as well as below threshold.

Taking into account that $\Psi_{1}(z)=\sqrt{\frac{2}{L}} \sin \frac{\pi z}{L}$, we obtain the singular contribution to the albedo from the first mode :

$$
\delta \alpha(q, t)=\frac{3 l}{\pi L}\left\{\frac{\exp \left\{Z_{1}(0) t\right\}-1}{\delta}+\frac{\exp \left\{Z_{1}(q) t\right\}-1}{\left\{\delta-\frac{D q^{2}}{\omega_{1}}\right\}}\right\}
$$

Below threshold the albedo is saturated. The peak at large times has a laplacian form $\propto \frac{1}{|\delta|+\frac{D q^{2}}{\omega_{1}}}$. At threshold and above there is narrowing of the peak with increasing time. Exactly at threshold the albedo is linear with time, and above threshold the albedo grows exponentially.

\section{Conclusions}

To summarize, superfluorescent emission of active photonic paint develops due to the cooperation of atoms along a diffusive trajectory through a system with cross-sectional dimensions of the order of a wavelength. The pulse therefore becomes narrower with decreasing mean free path of radiation until the cooperation number reaches its maximum value. The maximum cooperation number does not depend on disorder.

An external field enhances emission in backward direction. The peak sharpens in coherent backscattering during cooperative decay in a disordered system.

We thank A.V. Gol,tsev for useful suggestions. This work was supported by the Russian Fund for Fundamental Research under Grant number 97-0218078.

\section{References}

\section{References}


[1] Y. Kuga and A. Ishimaru, J.Opt.Soc.Am.A ,8, 831 (1984); M.P. van Albada and A. Lagendijk, Phys.Rev.Lett., 55, 2692 (1985); P.E. Wolf and G. Maret, Phys.Rev.Lett., 55, 2696 (1985).

[2] A.Z. Genack, in "Scattering and localization of classical waves in random media " P. Sheng (ed.), World Scientific, Singapore (1990).

[3] V. S. Letokhov, Zh. Eksp. Teor. Fiz. 53, 1442 (1967) [Sov. Phys. JETP 26, 835 (1968)].

[4] N.E. Ter-Gabrielyan, V.M. Markushev, V.R. Belan, C.M. Briskina, O.V. Dimitrova, V.F. Zolin, and A.V. Lavrov, Zh.Kvant.Elektr. 18, 313 (1991) [Sov. J.Quantum Electron. 21, 281 (1991)].

[5] C. Gouedard, D. Husson, C. Sauteret, F. Auzel, and A. Migus, J.Opt.Soc.Am. B 10, 2358 (1993).

[6] N. M. Lawandy, R. M. Balachandran, A. S. L. Gomes and E. Sauvain, Nature 368, 436 (1994).

[7] H. Taniguchi, S. Tanosaki, K. Tsujita, and H. Inaba, IEEE J.Quant.Electronics, 32, 1864 (1996).

[8] A. Yu. Zyuzin, Europhys. Lett. 26 (7), 517 (1994).

[9] D. S. Wiersma, M. P. van Albada and A. Lagendijk, Phys. Rev. Lett. 75, 1739 (1995).

[10] R.H. Dicke, Phys.Rev. 93,, 99 (1954).

[11] R. Bonifacio and L.A. Lugiato, Phys.Rev. A 11, 1507 (1975).

[12] R.Bonifacio, P.Schwendimann, and F.Haake, Phys.Rev.A 4, 302, 854 (1971).

[13] A.E. Siegman, Lasers, Oxford University Press, New York, (1986).

[14] A.A. Abrikosov, L.P. Gorkov, and I.E. Dzyaloshinski, Methods of Quantum Field Theory in Statistical Physics, Interscience New York (1963).

[15] F.T. Arecchi and E. Courtens, Phys.Rev. A 2, 1730 (1970) 
[16] A.V. Andreev, Yu.A. Il'inskii, and R.M. Khokhlov, Zh.Eksp.Teor.Fiz.73, 1296 (1977) [Sov.Phys. JETP46, 682 (1977)].

[17] E. Akkermans, P.E. Wolf, and R. Maynard, Phys.Rev.Lett.56, 1471 (1986). 